\title{
Un débat mouvementé
}

Une vue d'ensemble de la situation danoise

A lively debate. An overview of the status of human and social sciences in

Denmark

Un debate agitado. Una vista general de la situación de las ciencias humanas y sociales en Dinamarca

Jesper Eckhardt Larsen

Traducteur : Cécile Boyer et Michel Lussault

\section{CpenEdition}

\section{Journals}

Édition électronique

URL : http://journals.openedition.org/ries/561

DOI : 10.4000/ries.561

ISSN : 2261-4265

Éditeur

Centre international d'études pédagogiques

Édition imprimée

Date de publication : 1 décembre 2008

Pagination : 41-50

ISSN : 1254-4590

Référence électronique

Jesper Eckhardt Larsen, «Un débat mouvementé », Revue internationale d'éducation de Sèvres [En ligne], 49 | décembre 2008, mis en ligne le 01 décembre 2011, consulté le 20 avril 2019. URL : http:// journals.openedition.org/ries/561 ; DOI : 10.4000/ries.561 


\title{
Un débat mouvementé
}

\section{Une vue d'ensemble de la situation danoise*}

\author{
Jesper Eckhardt Larsen
}

Au Danemark, la tradition nationale en matière d'érudition humaniste et d'éducation publique en sciences humaines est forte. Le vocabulaire danois a adopté le concept des humaniora, de la Renaissance italienne, qui désigne les sciences (les plus) humaines, par opposition à la théologie et aux sciences naturelles. L'usage du concept de humaniora ressemble à celui fait du concept allemand de Geisteswissenschaften, mais avec une définition plus stricte. Cependant, le terme signifiant "la science», videnskab, est utilisé de manière aussi souple en danois que le terme Wissenschaft l'est en allemand, lorsqu'il est employé dans son sens le plus général. Cela rend donc l'expression «sciences humaines» (humanistisk videnskab) synonyme du mot humaniora. Les humanités (humaniora) ne sont donc pas directement liées au domaine artistique, comme c'est le cas dans le monde anglo-saxon, mais ont des visées savantes. Elles sont distinctes des sciences sociales et du droit. Les facultés de sciences humaines comprennent comme disciplines l'histoire (qui se divise en histoire classique, médiévale et moderne), la philosophie, la philologie (du danois et d'autres langues européennes), la rhétorique, la pédagogie, la littérature, l'histoire de l'art, la musicologie, les études cinématographiques, les études théâtrales, l'archéologie, l'ethnologie, etc. La psychologie et l'anthropologie sont des domaines de connaissances qui ont été récemment redéfinis dans les institutions comme appartenant aux sciences sociales. De nouvelles disciplines, telles que les études régionales ${ }^{1}$, l'étude des médias, les études de marketing culturel en lien avec le monde de l'entreprise et les études de langues sont parfois aussi considérées comme des études en sciences humaines, dites humanistiske studier. Notre analyse portera sur toutes ces disciplines, qu'on définira comme «sciences humaines» ou humaniora.

\section{UNE HISTOIRE INFLUENTE}

Si l'on se penche sur l'histoire politique de la connaissance au Danemark, on constate qu'une fracture s'est créée, à la fin du XIX ${ }^{\mathrm{e}}$ siècle, entre une culture universitaire élitiste et bourgeoise et une contre-culture rurale

\footnotetext{
* Article traduit par Cécile Boyer avec la collaboration de Michel Lussault.

1. Area studies: études à la croisée de plusieurs disciplines prenant en compte notamment la géographie, l'histoire, la culture, l'anthropologie, d'une zone donnée. Ex : la japanologie, les études européennes, etc. Voir : http://en. wikipedia.org/wiki/Area_studies/.
} 
incarnée par les idées enseignées dans les universités populaires ${ }^{2}$ (Folkehøjskoler). Le célèbre théologien luthérien et professeur N.F.S. Grundtvig (1783-1872) était lui-même issu de l'élite universitaire, mais il voua toute son énergie à critiquer sévèrement à la fois les écoles latines secondaires ${ }^{3}$ vouées aux humanités et l'université de Copenhague, bastions, selon lui, d'un savoir «mort». Il œuvra à l'inverse à l'élaboration, dans les universités populaires, d'un enseignement empreint de nationalisme romantique. Dès lors, une cassure, visible dans une certaine mesure jusqu'à ce jour, s'opéra au Danemark dans les fonctions et la nature des établissements chargés d'enseigner les sciences humaines. Il en résulta que l'enseignement des sciences humaines à l'université fut considéré comme le privilège d'une élite lettrée, alors que les universités populaires répondaient, quant à elles, à la demande de culture générale nationale du grand public.

Les transformations survenues au $\mathrm{XX}^{\mathrm{e}}$ siècle ont bien sûr fait évoluer le rôle des universités qui ont connu un phénomène de massification. Cela a réduit l'importance de la fonction jouée par les universités populaires dans l'éducation. Mais l'image négative ou limitative des universités qui sont décrites comme élitistes, purement scientifiques ou comme des écoles professionnelles a grandement influencé et influence encore aujourd'hui les discours tenus sur la place des sciences humaines dans l'enseignement supérieur danois. Pour beaucoup, l'enseignement supérieur a perdu sa fonction légitime d'éducation et ne sert qu'à former des bureaucrates et des professionnels dans des domaines spécialisés. On pourrait rétorquer que former un cercle d'intellectuels hautement qualifiés est essentiel dans une société démocratique. Mais les étudiants du second cycle universitaire qui se spécialisent en histoire, en philologie ou en philosophie ne sont en rien considérés par l'opinion publique comme des citoyens éclairés. La plupart du temps, on les considère plutôt comme un problème social, comme des gens pouvant potentiellement accroître les rangs des chômeurs, ou comme des profiteurs bénéficiant du système de financement public pour atteindre un certain épanouissement strictement personnel.

Alors que l'épanouissement personnel est une valeur très positive dans le monde de l'entreprise, il provoque souvent le rejet lorsqu'il s'agit de celui des étudiants en sciences humaines. En conséquence, les défenseurs des institutions chargées de l'enseignement des sciences humaines se retrouvent dans une position où ils n'ont guère d'autre choix que de mettre l'accent sur le fait que les savoirs qu'elles dispensent visent à former de futurs professionnels adaptés au monde du travail, sans quoi leur rôle dans l'enseignement public serait

2. Une université populaire est un organisme d'éducation, dont l'objectif est la transmission de savoirs théoriques et/ou pratiques pour tous.

3. Les collèges ou écoles latines étaient des établissements où l'on enseignait aux élèves les six classes latines, et les rudiments de la langue grecque. Ces écoles préparaient les élèves à entrer à l'université pour y faire leurs humanités. 
considéré comme négligeable. Comprendre ce contexte historique dans lequel les universités, attaquées, doivent se défendre, est d'une importance capitale pour saisir la teneur des débats actuels sur les sciences humaines au Danemark.

\section{L'ÉDUCATION DE BASE EN SCIENCES HUMAINES}

De tous les pays scandinaves, le Danemark est celui où la tradition d'enseigner la culture générale dans le secondaire a toujours perduré. En danois, ce concept d'acquisition de culture générale par les étudiants se dit almendannelse, ce qui correspond à la notion allemande d'Allgemeinbildung, britannique de liberal education et de «culture générale» en français. Depuis le XVIII ${ }^{\mathrm{e}}$ siècle, cette notion a joué un rôle fondamental, déterminant les objectifs et la raison d'être des sciences humaines dans les processus d'éducation. Dans les lycées danois (Gymnasier), le concept d'almendannelse contribue largement à définir les objectifs de ces trois années d'enseignement (Haue, 2008).

Le système éducatif danois ${ }^{4}$ suit le même modèle que les autres pays scandinaves : l'enseignement polyvalent. Cela comprend un minimum de neuf années d'enseignement obligatoire de niveau primaire et début du secondaire, dispensé soit dans des établissements publics (Folkeskole), soit dans des écoles privées. La loi ne stipule pas l'obligation d'assiduité scolaire, mais l'obligation pour les parents de donner à leurs enfants l'éducation nécessaire, que cet enseignement soit dispensé à la maison ou dans une institution scolaire. Les sciences humaines sont partie intégrante des programmes des écoles publiques. Des matières telles que la langue et la littérature danoise, l'histoire et les langues vivantes tiennent une grande place dans les neuf années d'enseignement obligatoire.

Après ce premier cycle, plus de $80 \%$ des élèves poursuivent leurs études. L'organisation des dernières années d'enseignement secondaire est divisée en deux types de cursus : le lycée classique, qui accueille presque $50 \%$ des élèves poursuivant leurs études, et d'autres établissements secondaires proposant des formations plus techniques ou plus tournées vers les métiers du secteur tertiaire. Le lycée classique, dont l'objectif est l'acquisition d'une solide culture générale, concourt largement à l'intérêt porté aux sciences humaines au Danemark. On pourrait avancer que cela explique les grandes différences qui existent entre la situation du Danemark et celle de la Suède. L'éducation secondaire suédoise fait entièrement partie des douze années d'enseignement obligatoire. Mais, dès les années 1960, le système éducatif secondaire suédois a été infléchi dans le sens de la prise en compte des perspectives d'emploi dans différents domaines spécifiques. Cela c'est traduit par une réduction de la place de l'enseignement des sciences humaines au profit de disciplines techniques ou tertiaires. Cela n'explique peut-être pas le faible niveau de fréquentation actuel des universités

4. Ndlr : Voir F. Rauser, «L’Éducation au Danemark », Revue internationale d'éducation de Sèvres nº 36, 2004. 
suédoises spécialisées en sciences humaines, mais cela renforce en tout cas indéniablement l'image d'une politique d'éducation résolument tournée vers l'emploi et vers les exigences économiques de la démocratie sociale suédoise.

Sur ce point, la situation de la Norvège se rapprocherait plus de celle du Danemark. Toutefois, avec les récentes réformes de l'organisation du secondaire instituant douze années d'enseignement polyvalent obligatoire, la Norvège se détache quand même de sa tradition continentale d'enseignement des sciences humaines au lycée, qui sont moins présentes qu'au Danemark. Ces politiques expliquent la relativement grande proportion d'étudiants en sciences humaines dans les universités danoises. Le Danemark est en effet à égalité en pourcentage avec les États-Unis et la France, avec environ 15 à $16 \%$ de ses étudiants suivant un cursus en sciences humaines ou un cursus dans un domaine artistique. Les pourcentages en Suède et en Norvège sont moitié moindres, avec respectivement $6 \%$ et $7 \%$ d'étudiants inscrits dans ces filières (Tertiaire de type A en 2005, OCDE).

\section{LA SITUATION DANS L'ENSEIGNEMENT SUPÉRIEUR}

Le Danemark fut l'un des premiers pays à introduire les réformes au niveau universitaire qui allaient conduire à l'instauration du Processus de Bologne dans l'espace européen à partir de 1999. Dès 1992, la structure en trois cycles de l'enseignement supérieur fut mise en place dans le système danois : une licence en trois ans, suivie d'un master en deux ans et d'un doctorat en trois ans. La précédente organisation de l'enseignement supérieur qui consistait en de longs cycles d'études spécialisées de cinq ans au minimum fut ainsi remplacée par un système qui offrait, pour les études supérieures en général, un choix limité de modules pendant la licence, et la possibilité de combiner une licence dans une discipline avec un master dans une autre. Le Danemark s'est en cela inspiré du cursus des États-Unis. Cependant, le modèle d'organisation de la licence danoise est dans l'ensemble plus proche de celui de la Grande Bretagne, car il laisse moins de choix dans les enseignements que la licence américaine (Larsen, 2006B).

Cela permet de séparer deux groupes d'étudiants. Le premier groupe est composé de ceux qui étudient deux disciplines, l'une en licence et l'autre en master. Les étudiants qui font ce choix seront aptes à enseigner en lycée. Les deux cursus suivis peuvent tous deux appartenir aux sciences humaines, ou non. Un étudiant peut ainsi devenir professeur de chimie et de danois, s'il a étudié ces deux disciplines en licence et en master. L'autre groupe est composé d'étudiants qui conservent la même discipline en licence et en master. Ces étudiants complètent ensuite leur formation par une année d'études supplémentaire où ils suivent des modules d'enseignement d'autres disciplines, le plus 
souvent dans d'autres matières en sciences humaines. Ces modules libres peuvent appartenir à n'importe quelle discipline, comme dans le modèle d'études américain. On peut donc en conclure que ces étudiants ont des profils plus spécialisés dans la discipline qu'ils ont principalement étudiée.

C'est à nouveau très différent de ce qui se passe en Suède. Les programmes universitaires suédois permettent aux étudiants de choisir des modules d'enseignement dans de nombreuses disciplines, ce qui fait que l'étudiant suédois en sciences humaines a un profil beaucoup plus généraliste que l'étudiant danois typique, car il étudie plus de matières différentes. Ce phénomène alimente certainement au Danemark le débat sur l'inadéquation de tels cursus universitaires au marché de l'emploi. En résumé : au Danemark, un diplômé en sciences humaines est presque toujours un spécialiste, rompu aux méthodes de recherche spécifiques à un domaine particulier des sciences humaines. La question est alors : que faire si cette personne ne trouve pas l'emploi correspondant exactement à ses compétences spécialisées sur le marché du travail? Un tel problème ne se poserait évidemment pas dans les pays où les sciences humaines n'interviennent qu'en tant que modules et non en tant que spécialités dans les programmes universitaires.

Le système danois explique qu'existe un débat politique sur le thème du «tout ou rien». Dans d'autres systèmes d'enseignement supérieur, la question pertinente est : les modules en sciences humaines peuvent-il contribuer de manière positive au développement personnel, professionnel et citoyen des individus? La réponse à une question posée en ces termes est évidemment : «oui». En Suède, le libre choix des modules d'enseignement explique le fait qu'il n'y ait que peu «d'humanistes purs» parmi les étudiants, et, si l'on se risque à une comparaison avec les États-Unis, il semble que ce libre choix de modules en sciences humaines soit une manière de sauver les filières en sciences humaines, qui s'appuie sur une conception de leur valeur éducative complémentaire (Larsen, 2006B). Au Danemark, la seule question qui focalise le débat est celle de l'adaptation au marché de l'emploi.

La problématique est donc la suivante : au Danemark, les cours en sciences humaines préparent-ils uniquement quelques heureux élus à l'enseignement et à la recherche? Ou existe-t-il aussi des cours de culture générale adressés au plus grand nombre? Il faut répondre à cela en deux temps. Dans l'enseignement secondaire, et spécialement au lycée, les sciences humaines dispensent une culture générale au plus grand nombre, avec des cours de langues, d'histoire et de philosophie de grande qualité. À l'inverse, au niveau universitaire, l'enseignement proposé est tourné vers la spécialisation : l'organisation des cursus est rigide et s'adresse à un public restreint qui se destine à devenir chercheur ou enseignant. Le nombre grandissant d'étudiants spécialisés en sciences humaines qui ne trouvent pas d'emploi conduit à ce que nombre d'acteurs politiques considèrent ces cursus comme inadaptés au monde actuel. 


\section{LES RAPPORTS ENTRE L'ACCÈS À L'EMPLOI ET LES SCIENCES HUMAINES}

Avant la massification de l'accès aux études supérieures dans les années 1960-1970, les facultés en sciences humaines s'adressaient uniquement à l'élite qui se destinait à une carrière universitaire, à ceux qui souhaitaient devenir des intellectuels indépendants (si leur niveau social le leur permettait), ou alors aux futurs enseignants du secondaire en lycée. Depuis vingt ans, la situation a beaucoup évolué. Aujourd'hui, seuls $25 \%$ des étudiants en sciences humaines deviennent enseignants en lycée et $25 \%$ chercheurs dans l'enseignement supérieur public ou privé. Les autres doivent donc trouver un emploi «nontraditionnel », compte tenu de leur cursus. Ce groupe d'étudiant a fait l'objet de multiples controverses, mais il faut reconnaître que la dynamique très favorable de l'économie danoise actuelle a progressivement éliminé les graves problèmes de chômage rencontrés auparavant par les diplômés en sciences humaines. En outre, l'absence d'intérêt pour ces profils dont les employeurs faisaient autrefois preuve a diminué, et l'on constate même un regain d'intérêt pour les compétences que les diplômés en sciences humaines détiennent.

Pourtant, malgré cette situation plus favorable, un âpre débat s'est engagé et persiste encore entre le ministère de la science, de la technologie et de l'innovation, la Confédération de l'industrie danoise (Dansk Industri) et la Chambre de commerce danoise (Dansk Erhverv) d'une part et, d'autre part, les communautés universitaires, les recteurs d'universités et leurs conseils d'administration. Le ministre de la science, de la technologie et de l'innovation a organisé, en 2005, trois groupes de réflexion indépendants constitués de représentants du marché du travail et d'universitaires pour évaluer l'utilité réelle des sciences humaines dans le monde du travail. Les trois groupes ont tiré les mêmes conclusions qui ont conforté le point de vue très utilitariste du ministre sur l'avenir des sciences humaines. Dans les discours tenus alors, l'argument clé est celui de l'économie et de l'emploi. Si les programmes d'enseignements en sciences humaines des universités ne répondent pas aux exigences du marché de l'emploi dans le secteur privé, alors ces filières devront être abandonnées. La contribution des sciences humaines à la société a ainsi été réduite de facto à leur contribution à l'économie. En conséquence, les programmes d'enseignements devront être tous réévalués dans cette optique au cours des prochaines années par une nouvelle entité dépendant du ministère : le conseil d'accréditation $A C E$ du Danemark. Et cet avenir n'est pas seulement promis aux programmes d'enseignements, mais s'appliquera aussi au domaine de la recherche.

Un tel sort n'est d'ailleurs pas réservé qu'aux sciences humaines, mais à toutes les champs de recherches et d'enseignement et ce spécialement depuis la Loi sur l'université danoise de 2003, qui a changé le mode de gestion des établissements. On est passé d'une gestion collégiale et démocratique à une 
structure pyramidale et centralisée dotant le ministère d'un pouvoir bien plus grand que jamais jusqu'alors. Cela confère au gouvernement une capacité de contrôle de l'enseignement et de la recherche universitaires sans précédent. Dans un tel contexte, l'opinion d'un ministre devient tout à coup décisive pour le développement des sciences humaines à l'échelle nationale.

En réaction à ce discours idéologique, six universités ont, en 2007, lancé une grande étude portant sur les carrières et l'expérience du marché du travail des diplômés en sciences humaines. Les conclusions de cette étude sont frappantes à plusieurs égards. Il en ressort qu'à ce jour, sur le marché du travail danois, les étudiants ayant choisi de poursuivre des études en sciences humaines ne sont pas désavantagés dans leur carrière professionnelle par rapport à ceux ayant fait d'autres types d'études (Humanistundersøgelsen, 2007). Malgré cela, l'orientation utilitariste persiste.

\section{LES ENJEUX COGNITIFS ET DISCIPLINAIRES}

Les universités danoises ont connu deux réformes majeures au cours des dix dernières années : d'abord, le Processus de Bologne de 1999, et ensuite la nouvelle Loi sur l'université danoise en 2003. La loi a modifié les modes de gestion des universités, nous venons de le souligner. Le Processus de Bologne a, quant à lui, eu un impact sur la conception de l'enseignement des sciences humaines. L'évolution de cette conception suit dans l'ensemble la tendance internationale, qui met aujourd'hui l'accent plus sur les compétences acquises par les élèves que sur le contenu et la progression scolaire. Pour les sciences humaines aussi, l'exigence de transférabilité des compétences acquises primet-elle? Ce n'est pas tant ce que l'on apprend qui compte, mais les compétences transférables qu'on acquiert; le savoir est un médium. Bien sûr, cela amoindrit la valeur du savoir, puisque son rôle principal n'est plus de transmettre les repères nécessaires pour affronter le monde moderne, de forger les consciences et de former à la citoyenneté.

Globalement l'enseignement des humanités n'a pas tant changé, mais l'image qu'on en a est différente. Aujourd'hui, on met en avant le fait que les spécialistes en sciences humaines sont des analystes très compétents et qu'ils sont capables de faire la synthèse de quantités d'informations pour en tirer le meilleur parti de manière organisée. Cette conception réductrice des aptitudes liées aux sciences humaines pose le même problème et conduit aux mêmes critiques que celles qu'on faisait au XIX ${ }^{\mathrm{e}}$ siècle à propos de l'apprentissage formel du latin : si l'on s'attache essentiellement aux méthodes, on peut faire abstraction du contenu de l'apprentissage. Et ainsi, un représentant de la Confédération de l'industrie danoise a pu déclarer qu'un spécialiste en sciences humaines pouvait toujours être remplacé par un autre, contrairement à un ingénieur ou à un économiste. 
Cette tendance à réduire le développement et à remettre en cause la légitimité de certaines disciplines est renforcée par la diffusion de l'idée que la recherche et l'enseignement sont guidés par les enjeux de société. Ce ne sont plus les disciplines en elles-mêmes mais les enjeux qui dictent les orientations à suivre pour la recherche et l'enseignement des sciences humaines. Cela a conduit au développement d'études appliquées : par exemple, les études en sciences humaines appliquées aux problématiques environnementales, ou à la relation homme/machine dans le domaine des technologies de l'information et de la communication, ou en médecine. Dans ce cas de figure, les sciences humaines amènent une "valeur ajoutée» aux autres domaines de recherches, comme les technologies de l'information et de la communication ou la médecine.

\section{DES ARGUMENTS VARIÉS SUR LE RÔLE DES SHS}

Si une approche restrictive de l'utilité sociétale des sciences humaines domine ces derniers temps le discours officiel, il n'en a pas toujours été ainsi. De 1945 à 1957, le discours tenu sur l'enseignement et la recherche en sciences humaines s'articulait autour de trois points : la paix, la culture et les valeurs. L'argument "pour la paix» faisait partie des préoccupations des grands acteurs internationaux comme l'UNESCO et les organismes apparentés dédiés aux sciences humaines. L'idée que les sciences humaines et leur étude promouvaient la paix découlait des suites de la guerre. L'argument «culturel» constituait une réminiscence du néo-humanisme qui a dominé la vie universitaire pendant la majeure partie du XIX ${ }^{\mathrm{e}}$ siècle. On considérait les études en sciences humaines comme un moyen de promouvoir la culture morale des nations. L'argument de la «valeur» s'apparentait aux deux autres et découlait directement de l'expérience du conflit mondial. On pensait que les sciences humaines étaient la sphère du règne des valeurs, et que, par conséquent, elles étaient d'une importance capitale pour aider les individus à faire les bons choix, souvent dans une perspective existentialiste.

De 1957 à 1968, la prise de conscience de la modernité nouvelle infléchit le discours tenu sur les sciences humaines. Le choc du lancement du Spoutnik fit découvrir au monde occidental qu'il avait été pris de vitesse par l'Union soviétique dans la course à la modernité. Quel était le rôle des sciences humaines - et en avaient-elles un à tenir dans le monde moderne? Un plaidoyer en faveur de la modernisation des sciences humaines grâce à l'empirisme se fit entendre. L'argument «scientifique» visait à transformer les sciences humaines en sciences exactes et prônait une réflexion «froide» sur le comportement psychologique et social de l'homme, afin de libérer la société de comportements traditionnels malsains.

Plus tard, le choc politique de 1968 se traduisit par une attaque politisée de la culture élitiste et bourgeoise. Les experts en sciences humaines 
n'étaient rien d'autre, pour les jeunes contestataires, que des dépositaires de l'idéologie bourgeoise qui devait être combattue pour établir une vision égalitariste de la culture. Cette idée se retrouva, un peu édulcorée, dans les politiques imposées aux conseils de recherche dans les humanités. On y avançait l'argument «social», qui découlait d'une nouvelle exigence d'utilité. Les humanistes devaient quitter leur tour d'ivoire et être utiles à la société, c'est-à-dire au peuple ou aux usagers.

Pendant les années 1980, l'aspect performatif gagna en force dans les discours tenus. On attendait de plus en plus des sciences humaines qu'elles apportent des solutions spécifiques à des problèmes spécifiques. L'argument de «résolution des problèmes» est résumé par cette formule : «de la recherche à la facture ${ }^{5}$. Les résultats des recherches menées devaient avoir une utilité concrète et pouvoir être applicables immédiatement en pratique. On peut citer comme exemple la linguistique de données ou la résolution des problèmes engendrés par la technologie. La nouvelle rhétorique argumentative employait les termes «diagnostic» et « remède». L’idée était que les spécialistes en sciences humaines savaient déceler les différents maux dont la société souffrait, et savaient trouver les remèdes pour l'en guérir.

Parallèlement, un revirement d'opinion sur la culture survint, et l'on revint à penser que la culture était primordiale dans le développement d'une société. Apparut l'argument de la «conscience culturelle» qui peut être vu comme un argument néo-humaniste. Il met en évidence le lien qui existe entre épanouissement personnel et socialisation culturelle. Cet argument était tombé en disgrâce dans les années 1970 à cause de sa connotation bourgeoise, mais il revint au premier plan dans les années 1980, parfois en tant que version postmoderne de l'individualisation. Les débats se teintèrent d'une dimension éducative. L'argument des «repères» consistait à affirmer que les sciences humaines, au lieu de poursuivre des objectifs utilitaires, devaient éduquer les étudiants pour leur permettre de trouver leurs repères dans un monde excessivement complexe et confus. Enfin, les pédagogues tentèrent de faire entendre l'idée que l'enseignement des sciences humaines devait s'adresser au plus grand nombre pour avoir une utilité éducative. On dit alors que l'argument de la «démocratisation» fit «sortir la recherche en sciences humaines de sa tour d'ivoire».

L'argument de la «diversité» apparut dans les années 1990. L’idée était qu'en étudiant la multitude de cultures passées et présentes, les sciences humaines contribuaient à la diversité de la réflexion dans la société. L'argument de la «démocratisation» des années 1980 tendit à devenir l'argument du «dialogue». Le chercheur ne devait pas faire pas de cours magistral à son auditoire, mais suggérer de possibles interprétations des expériences de la vie de tous les jours, dans un mouvement d'échange et de dialogue permanent avec le

5. Ce qui signifie que les chercheurs doivent pratiquement travailler à la commande. 
public concerné. Le lien entre la recherche et l'action est ici évident. Dans cette logique, la recherche avait la qualité de véhiculer une diversité de pensée, mais pas le privilège de détenir la vérité - c'est l'argument pragmatique.

Actuellement, les débats autour des ces différents argumentaires existent toujours dans la communauté des spécialistes. Pour le grand public, il existe une volonté politique de dispenser une culture générale au plus grand nombre d'élèves durant leurs années de primaire et de secondaire. Mais pour ce qui est des études supérieures, il semble que les sciences humaines soient menacées d'être réduites à leur plus simple expression. L'évolution des politiques universitaires en matière d'enseignement et de recherche est aujourd'hui guidée par de nouvelles exigences d'utilité inscrites dans le cadre général de l'économie du savoir et concernant en particulier tout ce qui touche à la vie professionnelle. Garder à l'esprit les arguments développés au fil des décennies dans les débats sur les sciences humaines, semblerait être la manière la plus féconde d'opérer mais ce n'est pas, semble-t-il, la façon dont les responsables danois envisagent les choses.

\section{BIBLIOGRAPHIE ET SOURCES}

LARSEN Jesper Eckhardt (2006A): «Ikke af brød alene... » argumenter for humaniora og universitetet $i$ Norge, Danmark, Tyskland og USA 1945-2005 ( Not on bread alone..." Argumentation for the Humanities and the University in Norway, Denmark, Germany and the United States, 1945-2005). Thèse de doctorat en danois, avec résumé en anglais. The Danish University of Education, Copenhagen.

LARSEN Jesper Eckhardt (2006B) : «The Role of the Humanities in the Bologna Idea of a University : Learning from the American Model?» in Revista Española de Education Comparada (El proceso de Bolonia. Dinámicas y deafios de la enseñanza superior en Europa a comienzos de una nueva época), vol. 12, pp. 309-327.

SARAUW Laura Louise (2008) : «Humanister på bestilling? Relationerne mellem humaniora og arbejdsmarked er under forandring. Men hvad med uddannelsesudviklingen?» (Des humanistes à la demande? Les relations entre les humanités et le marché du travail changent. Et en ce qui concerne le développement de l'éducation?) [Article en danois]. Voir : [http://adjunktportalen.hum.ku.dk/materialearkivet/].

HAUE Harry (2008): «Allgemeinbildung. Ein deutscher Begriff im dänischen Gymnasium 1750-2007 " (en allemand) University of Southern Denmark Studies in History and Social Sciences, vol. 360. University Press of Southern Denmark, Odense.

HAUE Harry (2007) : «Humaniora i det almene gymnasium» in Uddannelseshistorie 2007. Humanioras fødekcede - humanistisk viden $i$ videnssamfundet 41 . Yearbook of the Danish Society of the History of Education. S. Wiborg, L.R. Rasmussen, B. Larsen and J.E. Larsen (Eds.), Århus.

Humanistundersøgelsen 2007. Humanisternes veje fra uddannelse til job. Hovedrapport (Report on Humanistic Candidates 2007. The Way from Education to Employment for Humanists). [en danois] Voir : [http://www.ac.dk/files/pdf/Humanistundersoegelsen-2007.pdf] 